\title{
Toxina botulínica no tratamento de distonias faciais: avaliação da eficácia e da satisfação dos pacientes ao longo do tratamento
}

\author{
Botulinum toxin in the treatment offacial dystonia:evaluation of its efficacy and \\ patients'satisfaction along the treatment
}

\author{
Patrícia Gravito Costa ${ }^{1}$ \\ Líbia Aoki² \\ Fábio Perenes Saraiva ${ }^{3}$ \\ Suzana Matayoshi ${ }^{4}$
}

Departamento de Oftalmologia e Otorrinolaringologia da Faculdade de Medicina da Universidade de São Paulo (USP). São Paulo (SP).

${ }^{1}$ Médica residente de $3{ }^{\circ}$ ano do Departamento de Oftalmologia do Hospital das Clínicas da Faculdade de Medicina da Universidade de São Paulo (USP). São Paulo (SP).

${ }^{2}$ Médica estagiária do Departamento de Plástica Ocular do Hospital das Clínicas da Faculdade de Medicina da USP. São Paulo (SP).

${ }^{3}$ Médico residente de 3ำ ano do Departamento de Oftalmologia do Hospital das Clínicas da Faculdade de Medicina da USP. São Paulo (SP).

${ }^{4}$ Médica assistente do Departamento de Plástica Ocular do Hospital das Clínicas da Faculdade de Medicina da USP. São Paulo (SP).

Endereço de correspondência: Patrícia Grativol Costa. Rua Oscar Freire, 1702/38 - Pinheiros - São Paulo (SP) CEP 05409-011

E-mail: patriciagrativol@yahoo.com.br

Recebido para publicação em 06.10.2004

Versão revisada recebida em 14.02.2005

Aprovação em 04.03.2005

Nota Editorial: Após concluída a análise do artigo sob sigilo editorial e com a anuência do Dr. Marcos Carvalho da Cunha sobre a divulgação de seu nome como revisor, agradecemos sua participação neste processo.

\section{RESUMO}

Objetivos: Estudar a eficácia do tratamento com toxina botulínica nos pacientes com distonia facial e a satisfação com o tratamento ao longo do tempo. Métodos: Estudo retrospectivo de 42 pacientes portadores de distonia facial acompanhados no setor de Plástica Ocular da Clínica Oftalmológica do Hospital das Clínicas da Universidade de São Paulo. Resultados: Após as primeiras aplicações, 45,2\% dos pacientes deram notas entre 9-10 para melhora do espasmo, 35,7\% deram notas entre 7-8, $16,7 \%$ deram notas entre 5-6 e apenas um paciente deu nota 4 . Em relação ao intervalo de reaparecimento do espasmo, $4,8 \%$ dos pacientes referiram entre 5-6 meses, 64,2\% entre 3-4 meses e 31\% entre 1-2 meses. Ao longo do tratamento, $76,1 \%$ dos pacientes referiram manter a mesma nota sobre a melhora do espasmo, 19,1\% referiram melhora do resultado nas aplicações e apenas 4,8\% referiram piora da eficácia nas aplicações atuais. Quanto ao tempo de retorno do espasmo após aplicação, 64,2\% relataram não haver mudança ao longo do seguimento no serviço, $16,7 \%$ relataram aumento e $19,1 \%$ relataram diminuição do intervalo de remissão dos sintomas. Após aplicação, $19 \%$ dos pacientes apresentaram efeitos colaterais e 73,8\% dos pacientes referiram desconforto apenas leve ou moderado em relação à aplicação. Conclusões: $\mathrm{O}$ uso da toxina botulínica foi eficaz e não houve alteração da eficácia ao longo do tempo. São poucos os efeitos colaterais e boa tolerância à administração. É boa alternativa para melhorar a qualidade de vida desses pacientes evitando a cegueira funcional causada por essas doenças.

Descritores: Toxina botulínica tipo A/administração \& dosagem; Toxina botulínica tipo A/uso terapêutico; Espasmo hemifacial; Blefaroespasmo/terapia; Aceitação pelo paciente de cuidados de saúde; Satisfação do paciente; Recidiva

\section{INTRODUĈ̃̃O}

As distonias faciais mais freqüentes são o blefaroespasmo e o espasmo hemifacial. O blefaroespasmo é uma afecção adquirida das pálpebras, caracterizada por contrações tônicas espasmódicas do músculo orbicular, prócerus e corrugador dos supercílios. Durante muito tempo foi considerado como uma manifestação de natureza psiquiátrica. Atualmente acredita-se que seja uma doença orgânica neurológica, provavelmente relacionada a alterações nos gânglios da base ${ }^{(1-2)}$.

Ocorre principalmente em mulheres acima de 50 anos e geralmente é bilateral. Com o tempo, há progressão do quadro clínico, com aumento da 
freqüência de piscar e do tempo de oclusão palpebral durante o piscar, levando a dificuldades nas atividades diárias e até quadro de cegueira funcional.

O espasmo hemifacial é também uma doença caracterizada pela contração involuntária e intermitente dos músculos da face, geralmente unilateral. É presente mesmo durante o sono, diferente do blefaroespasmo.

A toxina botulínica mostra-se benéfica nos estudos duplocego de pacientes com blefaroespasmo e síndrome de Meige, e tem sido o tratamento de escolha para o blefaroespasmo. No espasmo hemifacial também tem demonstrado resultados satisfatórios ${ }^{(3-5)}$.

O objetivo deste trabalho é estudar a eficácia da toxina botulínica nos pacientes com blefaroespasmo essencial e espasmo hemifacial, assim como avaliar a satisfação desses pacientes com o tratamento ao longo do tempo.

\section{MÉTODOS}

Estudo retrospectivo, com aplicação de questionário, onde foram incluídos 42 pacientes do setor de Plástica Ocular do Hospital das Clínicas da Universidade de São Paulo (HCFMUSP). Estes pacientes faziam aplicação periódica de toxina botulínica e estavam em acompanhamento ambulatorial em um período que variava de 8 meses a 10 anos.

O grupo era constituído por 11 homens e 31 mulheres, com idade variando de 45 a 85 anos. Vinte e três pacientes apresentavam diagnóstico de blefaroespasmo essencial, 17 pacientes de espasmo hemifacial e 2 pacientes de síndrome de Meige. O tempo de início do espasmo variou de 2 a 30 anos. A dose aplicada foi 12,5 unidades de toxina botulínica tipo A (Botox ${ }^{\circledR}$ ) ao redor de cada olho, 2,5 unidades por ponto de aplicação (diluição da toxina: $100 \mathrm{u}$ Botox $^{\circledR} / 4 \mathrm{ml} \mathrm{NaCl} 0,9 \%$ ). Dependendo da intensidade das contrações foram feitas aplicações na região dos músculos prócerus e corrugador do supercílio (Figura 1).
Foi aplicado um questionário aos pacientes, que constava das seguintes perguntas:

1) Em relação ao espasmo, dê uma nota de 0-10 quanto à melhora após aplicação;

2) Quanto tempo demorou a retornar os sintomas (espasmo)?;

3) Ao longo do tempo de tratamento, houve mudança em relação ao resultado da aplicação? (em relação à eficácia? em relação ao tempo de reaparecimento dos sintomas?);

4) Houve algum efeito colateral durante o tratamento?;

5) Como você classificaria o desconforto da aplicação?

\section{RESULTADOS}

Com relação à resposta ao tratamento com a toxina botulínica ao longo das aplicações, 45,2\% dos pacientes $(n=19)$ deram notas entre 9-10 para melhora do espasmo após as primeiras aplicações, 35,7\% deram notas entre 7-8 $(n=15)$, $16,7 \%$ dos pacientes deram notas entre 5-6 $(n=7)$ e apenas um paciente deu nota 4 para melhora do espasmo $(2,4 \%)$. Não houve nenhuma nota abaixo de 4 para a melhora do espasmo (Gráfico 1).

Em relação às primeiras aplicações, 4,8\% dos pacientes referiram tempo de remissão dos sintomas entre 5-6 meses $(n=2), 64,2 \%$ entre $3-4$ meses $(n=27)$ e $31 \%$ referiram necessidade de nova aplicação entre 1-2 meses $(n=13)$ (Gráfico 2).

Considerando apenas os pacientes com blefaroespasmo e síndrome de Meige $(n=25), 60 \%(n=15)$ referiram tempo de remissão entre $3-4$ meses e $40 \%(n=10)$ entre $1-2$ meses. Nenhum paciente relatou tempo de remissão entre 5-6 meses. Entre os pacientes com espasmo hemifacial $(n=17), 11,8 \%$ $(\mathrm{n}=2)$ responderam tempo de remissão entre 5-6 meses, 70,6\% $(n=12)$ responderam entre $3-4$ meses e $17,6 \%(n=3)$ entre $1-2$ meses (Quadro 1).

Comparando as aplicações iniciais com as subseqüentes, $76,1 \%(\mathrm{n}=32)$ dos pacientes relataram manter a mesma nota
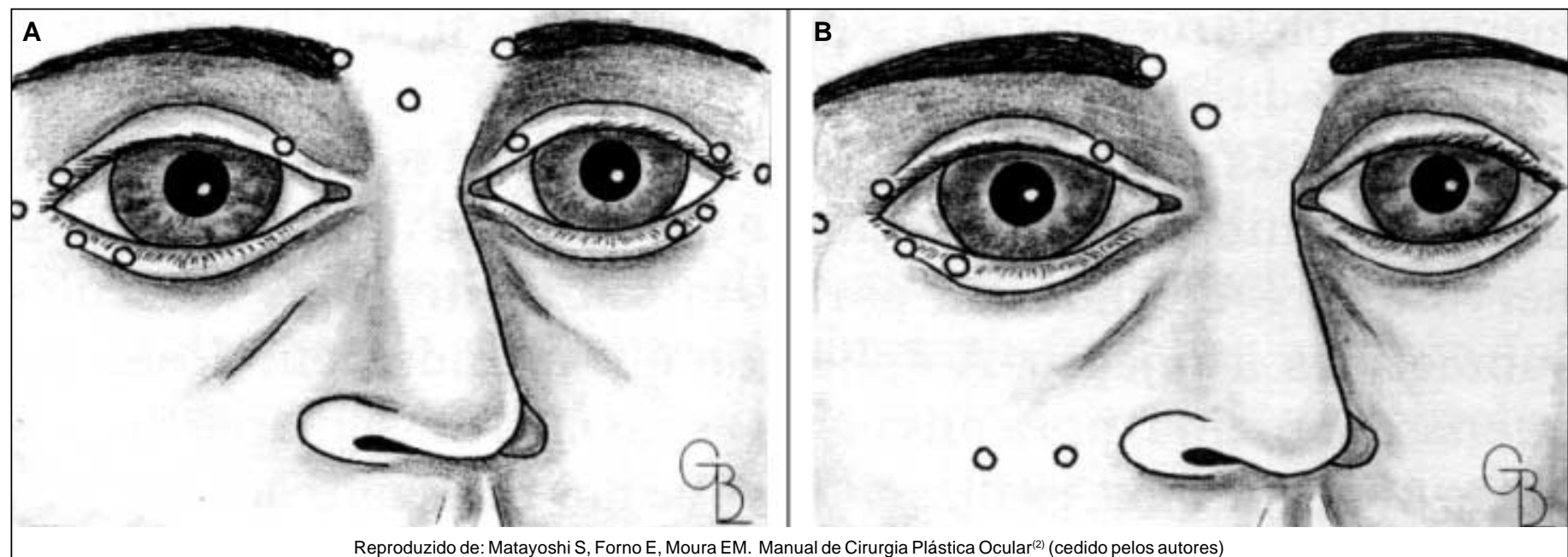

Reproduzido de: Matayoshi S, Forno E, Moura EM. Manual de Cirurgia Plástica Ocular ${ }^{(2)}$ (cedido pelos autores)

Figura 1 - Locais de aplicação da toxina botulínica no blefaroespasmo essencial (A) e no espasmo hemifacial (B). 
sobre a melhora do espasmo, $19,1 \%$ referiram melhora do resultado nas aplicações atuais $(n=8)$ e apenas $4,8 \%$ referiram piora da eficácia nas aplicações atuais $(n=2)($ Gráfico 3$)$.

Quanto à duração do efeito da toxina botulínica entre as aplicações no início do tratamento e as aplicações atuais, $64,2 \%$ relataram não haver mudança no tempo de retorno do espasmo após aplicação $(\mathrm{n}=27), 16,7 \%$ dos pacientes relataram aumento no tempo de remissão dos sintomas $(n=7)$ e $19,1 \%$ dos pacientes relataram diminuição do intervalo de remissão dos sintomas ao longo do seguimento no serviço $(\mathrm{n}=8)$ (Gráfico 4).

Os pacientes também foram questionados a respeito de efeitos colaterais após a aplicação, como ptose, olho seco, epífora, ectrópio, entrópio e diplopia. Dos 42 pacientes, 19\% apresentaram efeitos colaterais, sendo que o mais comum foi

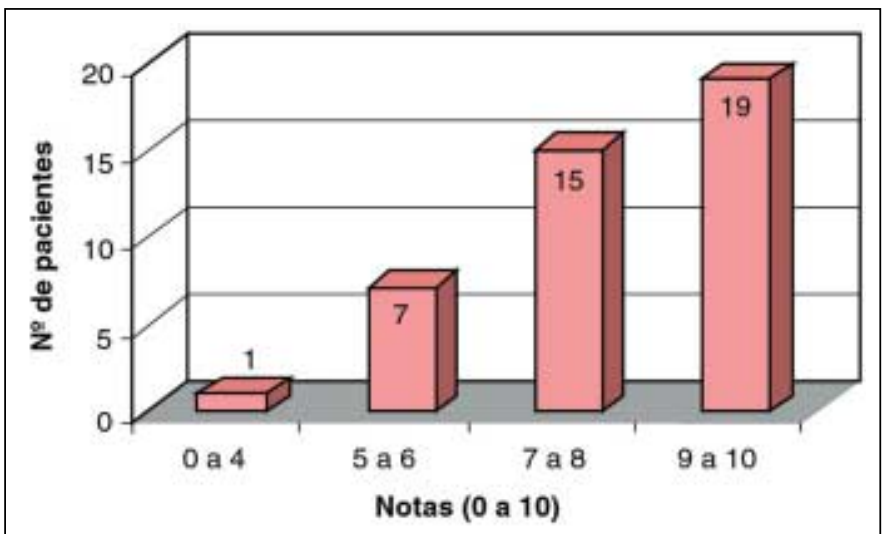

Fonte: Pacientes do ambulatório de oftalmologia do HC-FMUSP

Gráfico 1 - Notas dadas pelos pacientes de acordo com a melhora do espasmo após as primeiras aplicações

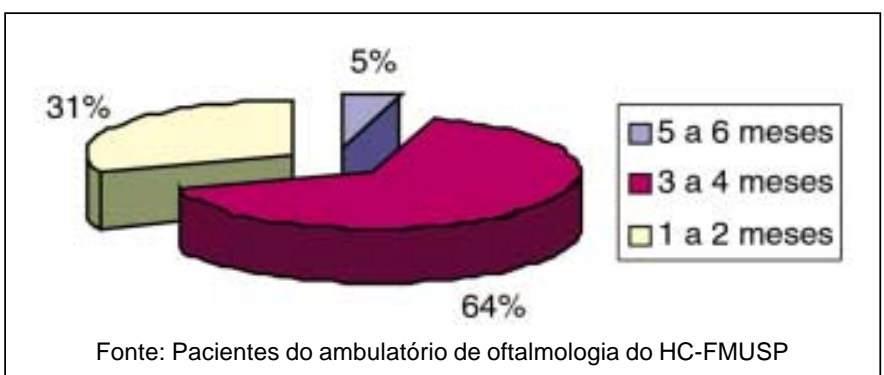

Gráfico 2 - Tempo de reaparecimento do espasmo após as primeiras aplicações de toxina botulínica

\begin{tabular}{|lrc|}
\hline $\begin{array}{l}\text { Quadro 1. Tempo de remissão do espasmo separado por grupos } \\
\text { de moléstias }\end{array}$ \\
$\begin{array}{l}\text { Tempo de } \\
\text { remissão }\end{array}$ & $\begin{array}{c}\text { Blefaroespasmo } \\
+ \text { Meige }\end{array}$ & $\begin{array}{c}\text { Espasmo } \\
\text { hemifacial }\end{array}$ \\
$5-6$ meses & $0 \%(n=0)$ & $11,8 \%(n=2)$ \\
$3-4$ meses & $60 \%(n=15)$ & $70,6 \%(n=12)$ \\
$1-2$ meses & $40 \%(n=10)$ & $17,6 \%(n=3)$ \\
Fonte: Pacientes do ambulatório de oftalmologia do HC-FMUSP
\end{tabular}

ptose palpebral (14,3\%). Com relação ao desconforto da aplicação, 73,8\% dos pacientes relataram desconforto apenas leve ou moderado, referindo estarem satisfeitos com o tratamento.

\section{DISCUSS ÃO}

A toxina Botulínica tipo A (TBA) é uma neurotoxina produzida pela bactéria Clostridium botulinum. Ela age paralisando a ação muscular através do bloqueio da transmissão colinérgica nos terminais pré-sinápticos. Ela foi aprovada para o tratamento do estrabismo e do blefaroespasmo, logo sendo indicada para o tratamento de diversas desordens associadas à excessiva contração muscular, incluindo distonia cervical, distonia de membros, blefaroespasmo e espasmo hemifacial ${ }^{(5)}$.

As distonias faciais podem causar uma cegueira funcional, justificando a investigação para o tratamento dessas afecções. Estudos mostram que a TBA é efetiva em melhorar o espasmo e a qualidade de vida dos pacientes, apesar de não representar uma cura definitiva ${ }^{(6)}$.

Neste trabalho, $81 \%$ dos pacientes deram notas maiores ou iguais a 7 para a melhora dos sintomas, o que está de acordo com a literatura, que mostra taxas de sucesso entre 69 e $100 \%$ com o tratamento ${ }^{(6)}$.

A duração do efeito da toxina botulínica variou entre 3-4 meses em $64,2 \%$ dos pacientes, necessitando nova aplicação após este período. Separando os pacientes por grupos de moléstias (Quadro 1), nota-se uma diferença entre o tempo de remissão

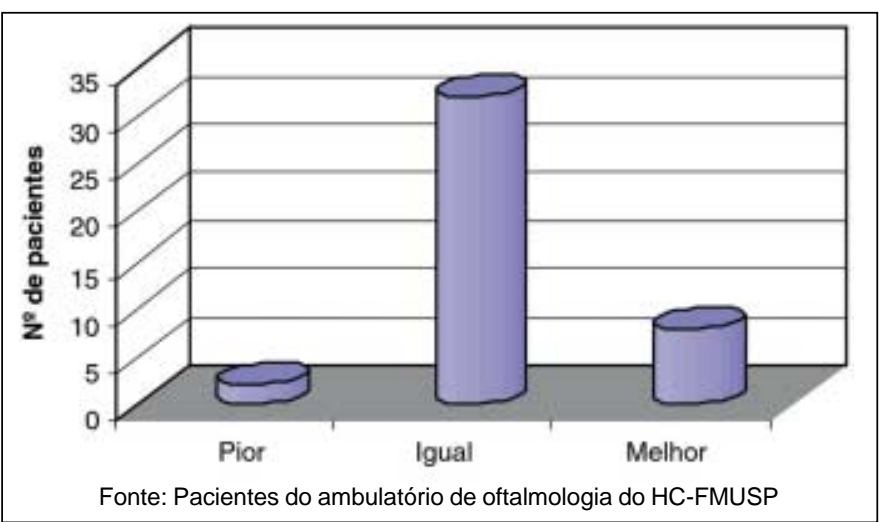

Gráfico 3 - Avaliação da eficácia do tratamento após sucessivas aplicações

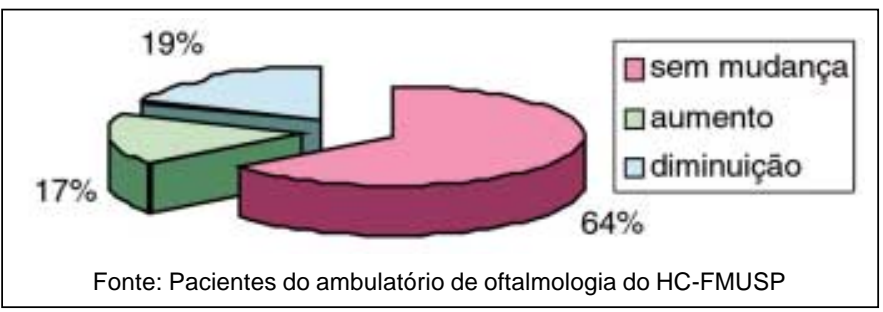

Gráfico 4 - Alteração do tempo de reaparecimento do espasmo após sucessivas aplicações 
do espasmo entre os dois grupos, sendo maior nos pacientes com espasmo hemifacial, como é descrito na literatura ${ }^{(7)}$.

Em relação à melhora dos sintomas e duração do efeito da TBA após injeções repetidas, os trabalhos na literatura são discordantes. Existem dúvidas se a aplicação repetida de TBA poderia causar tolerância no indivíduo ao longo do tempo. Alguns autores ${ }^{(8)}$ relatam em seu estudo que foi necessário aumentar a dose da TBA em 50\% para manter os 3 a 4 meses de alívio dos sintomas ao longo de injeções repetidas. Uma das hipóteses para esse declínio da eficácia seria devido à produção de anticorpos contra a TBA ${ }^{(9)}$. Quanto menor o intervalo entre as aplicações, mais precocemente ocorreria formação desses anticorpos.

Outros trabalhos, no entanto, não observaram mudança em relação à eficácia ou necessidade de aumento da dose da medicação no seguimento em longo prazo ${ }^{(10-13)}$. Por outro lado, a manutenção do tratamento periódico poderia diminuir a dose necessária para controle do espasmo facial. No presente estudo, $76,1 \%$ dos pacientes relataram que continuaram apresentando a mesma melhora do espasmo e $64,2 \%$ relataram o mesmo período de remissão dos sintomas após aplicações repetidas, estando de acordo com os trabalhos acima citados.

Observa-se também que a administração da TBA é bem tolerada. Quanto aos efeitos colaterais, 19\% dos pacientes apresentaram alterações transitórias, como ptose palpebral, ectrópio de pálpebra inferior e diplopia. O mais comum foi a ptose palpebral temporária, o que está de acordo com a literatura $^{(6)}$. Como a população envolvida tem perfil diferente daqueles que fazem aplicação da toxina estética, a ptose palpebral é bem aceita em virtude da transitoriedade e do conforto pela melhora do espasmo. Além disso, 73,8\% dos pacientes referiram desconforto leve a moderado em relação à aplicação, relatando satisfação com o tratamento.

\section{CONCLUSÕES}

Concluímos que não houve alteração da eficácia da TBA, com aplicações sucessivas, tanto em relação à melhora do espasmo quanto em relação ao tempo de reaparecimento dos sintomas. A terapia é bem aceita pelos pacientes que continuam fiéis ao tratamento ao longo do tempo e é boa a tolerância à administração. Representa uma boa alternativa para melhorar a qualidade de vida desses pacientes evitando a cegueira funcional causada por essas moléstias.

\section{ABSTRACT}

Purposes: To study the efficiency of botulinum toxin treatment in facial dystonia patients and their satisfaction along treatment. Methods: Retrospective study of 42 facial dystonia cases followed at the Oculoplastic Surgery Department of the "Hospital das Clínicas" of the University of São Paulo. Results: Following the first injections, $45.2 \%$ of the patients scored the improvement of the spasms between 9-10, 35.7\% scored between 7-8, 16.7\% between 5-6 and only one patient scored the improvement of the spasm as 4 . According to the remission time of the symptoms, $4.8 \%$ of the patients reported between 5-6 months, $64.2 \%$ between 3-4 months and $31 \%$ reported the need for a new injection after 1-2 months of the previous injection. During treatment, $76.1 \%$ of the patients maintained the same score for spasm improvement after the botulinum toxin injection, $19.1 \%$ reported improvement and only $4.8 \%$ noted worsening of efficiency of the last injections. Along the follow-up, $64.2 \%$ reported no change in the time of return of the symptoms, $16.7 \%$ had increased and $19.1 \%$ had decreased remission time of the symptoms. After injections, $19 \%$ of the patients presented side effects and $73.8 \%$ of the patients complained of mild to moderate discomfort during the injection. Conclusions: Botulinum toxin was efficient and no change of efficiency along the time was observed. Side effects are few and the tolerance to injections are good. It is a good alternative in order to improve quality of life of these patients avoiding functional blindness caused by these diseases.

Keywords: Botulinum toxin type A/administration \& dosage; Botulinum toxin type A/therapeutic use; Hemifacial spasm; Blepharospasm/therapy; Patient acceptance of health care; Patient satisfaction; Recurrence

\section{REFERÊNCIAS}

1. Gonnering RS. Blepharospasm and hemifacial spasm. In: Dortzbach RK. Ophthalmic plastic surgery: prevention and management of complications. New York: Raven Press; 1994. p.141-55.

2. Matayoshi S, Forno E, Moura EM. Blefaroespasmo essencial. In: Nano-Costa M, Schellini AS, Moura EM. Manual de cirurgia plástica ocular. São Paulo: Roca; 2003. p.127-35.

3. Carvalho RML, Gomi CF, Matayoshi S, Moura EM. Tratamento do blefaroespasmo e distonias faciais correlatas com toxina botulínica - estudo de 16 casos. Arq Bras Oftalmol. 2003;66(1):13-7.

4. Machado FCN, Fregni F, Campos CR, Limongi JCP. Espasmo hemifacial bilateral. Relato de caso. Arq Neuropsiquiatr. 2003;61(1):115-8.

5. Cunha CC, Aguirre PA, Souza Dias CR. Tratamento do espasmo facial unilateral com toxina botulínica tipo A. Arq Bras Oftalmol. 1998;61(1):54-60.

6. Tucha O, Naumann M, Berg D, Alders GL, Lange KW. Quality of life in patients with blefaroespasm. Acta Neurol Scand. 2001;103(1):49-52.

7. Price J, O'Day J. Efficacy and side effects of botulinum toxin treatment for blepharospasm and hemifacial spasm. Aust N Z J Ophthalmol. 1994;22(4):255-60.

8. Jitpimolmard S, Tiamkao S, Laopaibonn M. Long term results of botulinum toxin type A (Dysport) in the treatment of hemifacial spasm: report of 175 cases. J Neurol Neurosurg Psychiatry. 1998;64(6):751-7.

9. Snir M, Weinberger D, Bourla D, Cristal-Shalit O, Dotan G, Axer-Siegel R. Quantitative changes in botulinum toxin a treatment over time in patients with essential blefarospasm and idiophatic hemifacial spasm. Am J Ophthalmol. 2003;136(1):99-105.

10. Dressler D. Clinical features of antibody-induced complete secondary failure of botulinum toxin therapy. Eur Neurol. 2002;48(1):26-9.

11. Defazio G, Abbruzzese G, Girlanda P, Vacca L, Curra A, De Salvia R, et al Botulinum toxin a treatment for primary hemifacial spasm: a 10-year multicenter study. Arch Neurol. 2002;59(3):418-20.

12. Drummond GT, Hinz BJ. Botulinum toxin for blefarospasm and hemifacial spasm: stability of duration of effect and dosage over time. Can J Ophthalmol. 2001;36(7):398-403.

13. Ainsworth JR, Kraft SP. Long-term changes in duration of relief with botulinum toxin treatment of essential blefarospasm and hemifacial spasm. Ophthalmology. 1995;102(12):2036-40. 